City University of New York (CUNY) CUNY Academic Works

\title{
Language Complexity, Belief-consistency and the Evaluation of Policies
}

Matthew H. Goldberg

Yale University

Cheryl L. Carmichael

CUNY Brooklyn College

\section{How does access to this work benefit you? Let us know!}

More information about this work at: https://academicworks.cuny.edu/bc_pubs/241

Discover additional works at: https://academicworks.cuny.edu

This work is made publicly available by the City University of New York (CUNY).

Contact: AcademicWorks@cuny.edu 
Language Complexity, Belief-consistency, and the Evaluation of Policies

Matthew H. Goldberg and Cheryl L. Carmichael

Brooklyn College \& Graduate Center, City University of New York

[Word count: 6235 (main text) +133 (abstract)] 


\begin{abstract}
Policy proposals often contain complex legal, technical, or scientific jargon making it difficult for people to evaluate their favorability towards the policy. We proposed one experiment testing the effect of language complexity on people's evaluation of a policy proposal as moderated by their initial policy beliefs. We hypothesized that when a policy was consistent with one's beliefs or if participants had no policy preference, they would evaluate it more favorably when it was simple than when it was complex; when a policy was inconsistent with one's beliefs, they would evaluate it less unfavorably when it was complex than when it was simple. Results confirmed our hypotheses. This demonstrates that complex information does not always make people judge policies more negatively but rather causes people to weigh complex information less heavily in their judgments. Keywords: processing fluency, language complexity, policy attitudes, attitudes
\end{abstract}




\section{Language Complexity, Belief-consistency, and the Evaluation of Policies}

In current American politics, it is extremely difficult for the average citizen to evaluate policy proposals because they often contain complex legal, technical, or scientific jargon. Although any policy proposal may contain complex language, some issues are especially prone to complex language use because they consist of complicated economic, technological, or scientific concepts. Such issues include taxes and environmental conservation policies. Complex policy language is problematic for American voters because it can interfere with their ability to discern whether a message is consistent or inconsistent with their beliefs. The current experiment will test the proposition that as issue language complexity increases, people will mistakably deviate from their own policy preferences by rating preference-consistent policies less favorably and preference-inconsistent policies less unfavorably than they otherwise would. The findings from this experiment will help explain why Americans may vote against their own policy preferences, pointing to the importance of policy communication and civic education.

\section{Issue Language Complexity}

In order for citizens to vote according to their policy preferences, they have to understand the corresponding policy proposals. Yet, research shows that most voters agree that ballot initiatives are too complex to understand (Cronin, 1989). Therefore it is not surprising that citizens are less likely to participate when they must vote for legislation (which may be difficult to understand) rather than for a representative (Cronin, 1989; Everson, 1981). Magleby (1984) argues that the lack of voting on ballot initiatives can be explained by the use of overly complex language. Thus, even for people who 
participate, complex language makes it difficult for them to accurately gauge the extent to which the policy lines up with their existing beliefs about whether or not the policy should be implemented.

The research examining language complexity, also known as processing fluency, has a very clear conclusion: information that people can easily process (e.g. simple language) is judged more favorably than information that is difficult to process (e.g., complex language; for a review, see Reber et al., 2004). Although processing fluency has been manipulated both perceptually (e.g. small font; Gervais \& Norenzayan, 2012) and conceptually (e.g. complex language; Shockley \& Fairdosi, 2015; Shepherd \& Kay, 2012), we focus on fluency as defined by the ease with which language is processed. This is because of the clear relevance complex language has to the evaluation of policy proposals.

In a series of experiments, Oppenheimer (2006) manipulated the language complexity of three different kinds of text (personal statements, dissertation abstracts, and philosophical essays) and had participants rate the intelligence of the author. In all experiments, increasing complexity produced lower intelligence ratings (Oppenheimer; 2006; Experiments 1-3). The effect remained robust regardless of the actual quality of the essay, or prior beliefs about the quality of the essay. Oppenheimer demonstrates that such effects are at least partly driven by how easily people can process the information (i.e. fluency). That is, complex language made the information difficult to process and therefore affected people's judgment of the supposed authors.

The effect of fluency on stock market prices provides a compelling real-world example. Alter and Oppenheimer (2006) found that companies with fluent names 
performed better in two U.S. stock markets than companies with relatively less fluent names.

Recent experimental evidence showed, compared to a simply worded policy, a complexly worded policy reduced people's likelihood of voting for that policy and led to less favorable attitudes toward the policy (Shockley \& Fairdosi, 2015). This finding appears to be robust to whether participants agree or disagree with the underlying message of the policy. In addition to reducing people's likelihood of voting for a policy, another series of studies found that complex language motivates people to avoid learning more about the issue - an effect termed "the perpetuation of ignorance" (Shepherd \& Kay, 2012).

Based on such evidence, it would appear that complex language always leads people to judge information less favorably. However, a more precise analysis suggests language complexity determines the extent to which people rely on the content of the message to inform their attitudes. That is, when the content of a message is simple and easy to comprehend, people judge the message based on its merit (i.e., they take the central route to persuasion) rather than on peripheral cues, such as the status of the source (Petty \& Cacioppo, 1986; Hafer, Reynolds, \& Obertynski, 1996). When the content of a message is complex, on the other hand, people rely more on peripheral cues to form their judgments. For example, Hafer, Reynolds, and Obertynski (1996), found that participants rated a simply worded message more favorably when it was strong than when it was weak, meaning that they judged the message based on its merit. When the message contained complex language, participants rated it more favorably when it came from a high-status source than a low-status source, but the strength of the message had no effect 
on participants' judgment of it. This supports the idea that, when the message language is simple, people rely more on message content (e.g. whether one agrees or disagrees with it) to inform their judgments but rely less on message content when the message language is complex.

Accumulating evidence suggests that, rather than being positive itself, easily processed information carries more weight in people's judgments than information that is difficult to process. For example, Shah and Oppenheimer (2007) presented participants with conflicting (both positive and negative) information about an object and manipulated whether the positive information was easy to process (low complexity) and negative information difficult to process (high complexity), or vice versa. Participants judged the object more positively when the positive information was relatively easier to process but judged it more negatively when the negative information was relatively easier to process. In other words, information that was easy to process had a greater impact on people's judgments.

The current experiment builds on previous research (Shah \& Oppenheimer, 2007; Shockley \& Fairdosi, 2015) by considering how people's pre-existing policy preferences alter the effects of language complexity on policy favorability ratings. As opposed to using positive and negative information (Shah \& Oppenheimer, 2007), the current experiment will demonstrate that people's pre-existing policy preferences will determine how language complexity affects their judgment of a policy proposal. Our proposed mechanism is the same as previous research in this domain (Shockley \& Fairdosi, 2015). That is, complex language makes information difficult to process and therefore affects judgment of the attitude object. However, rather than arguing that difficult processing 
always leads to more negative judgments, we instead argue that it makes people weigh the information less heavily (Shah \& Oppenheimer, 2007). For example, relative to simple language, complex language makes it difficult for people to process their agreement with a preference-consistent message or their disagreement with a preferenceinconsistent message. That is, people's judgments should be more extreme when the policy is in simple language because it is easier for people to process their agreement or disagreement with the policy. On the contrary, complex language makes processing difficult and should therefore lead to less extreme ratings (i.e. preference-consistent policies less positive and preference-inconsistent policies less negative). This would suggest that the influence of language complexity depends on whether or not one agrees with the policy.

We will extend the existing findings by manipulating whether or not a policy is consistent with participants' pre-existing policy preferences (consistent vs. inconsistent vs. no preference) in addition to manipulating issue language complexity (simple vs. complex) to test the hypothesis that people will judge a preference-consistent policy more favorably when it is simple than when it is complex and will judge a preferenceinconsistent policy less unfavorably when it is complex than when it is simple. This is because a simple preference-inconsistent policy can more easily be processed as inconsistent with one's current beliefs than a complex one. Likewise, preferenceconsistent information should be more easily processed and thus more likely to produce a positive judgment when it is simple rather than when it is complex. When people have no policy preference, we expect them to judge the policy more favorably when it is simple than when it is complex (Shockley \& Fairdosi, 2015). Because we expect the effect of 
complexity to reverse depending on whether the policy is consistent or inconsistent with the participant's initial policy preference, we do not expect a main effect of complexity.

We do, however, expect a main effect of policy preference such that preference-

consistent policies will be rated more favorably than preference-inconsistent policies (e.g. Edwards \& Smith, 1996; See Figure 1).

\section{Method}

\section{Participants and Power Analysis}

Our preregistration can be found at https://osf.io/mrkv3/. ${ }^{1}$ Three hundred and twenty seven participants $\left(M_{\text {age }}=35.82, S D_{\text {age }}=11.50 ;\right.$ Male $=179$, Female $=146$, Did not respond $=2$ ) were recruited from Amazon's Mechanical Turk (mTurk). The study was restricted to participants in the United States who had completed a minimum of 100 tasks, and had a task approval rate of at least $85 \%$.

We used the $\mathrm{G}^{*}$ Power software to calculate our sample size (for details on the G*Power software, see Faul, Erdfelder, Buchner, \& Lang, 2009; See Appendix A for all information we entered into $G^{*}$ Power). The methods used in the current experiment closely mirror those of Shockley and Fairdosi (2015), which enabled an informed effect size estimate to calculate a sufficient sample size. They found effects of complexity on policy attitude with sizes ranging from Cohen's $d=.29$ to $d=.44$. Although we did not expect the 2 (Language complexity: Simple vs. Complex) X 3 (Preference-consistency:

\footnotetext{
${ }^{1}$ The Open Science Framework (OSF) preregistration at https://osf.io/mrkv3/ contains our primary hypothesis of interest and the procedure used for the study. However, after receiving helpful reviewer feedback, we made several additions to the analysis plan and to the procedure without deviating from the analyses and procedure that were planned in the preregistration. Additionally, we modified our goal sample size to accommodate the additional analyses. Because all hypotheses, analyses, and procedures were approved before data collection (as per the rules of registered reports) we mark such analyses as preregistered even though they contain additional information than what was included in the OSF preregistration. A detailed explanation of the reasons for making adjustments after completing the preregistration, as well as the analysis plan that was approved in stage 1 of this manuscript before data collection, can be found at https://osf.io/dt2u2/.
} 
Consistent, Inconsistent, or No preference) interaction to be moderated by issue, we calculated our goal sample size to retain at least $80 \%$ power to test the 2 (Language complexity: Simple vs. Complex) X 3 (Preference-consistency: Consistent, Inconsistent, or No preference) X 2 (Issue: Climate Change vs. Taxing Corporations) interaction with an effect size of $d=.365$ (i.e. the average effect size in the range above). This power analysis calls for 293 participants. Using a sample size that has adequate power to detect the above three-way interaction ensured that we had more than adequate power to detect the 2 (Language complexity: Simple vs. Complex) X 3 (Preference-consistency: Consistent, Inconsistent, or No preference) interaction, which was our primary analysis of interest.

Because we expected to exclude participants for failing to pass the attention check (Hauser \& Shwarz, 2016), we recruited 327 participants in order to retain adequate statistical power after excluding such participants. Participants passed the attention check at a higher rate than expected: 6 participants $(1.8 \%)$ were excluded for failing it (4 in the simple condition, 2 in the complex condition). One additional participant was excluded for failing to report their position on corporate taxes. To further ensure an appropriate sample size, we set our minimum cell sample size to 15 participants for our primary groups of interest (i.e. not including the no-preference category). We surpassed the minimum cell sample size; the smallest cell sample size was 25 .

\section{Procedure}

All materials and procedures were exactly as preregistered. First, participants read an information page and complete a modified version of Zhou and Fishbach's (2016) remedy to participant attrition in which participants typed a sentence stating they will 
complete the full survey. Next, participants indicated their position on two political issues (Climate Change or Taxing Corporations) and answered demographic questions.

Participants were then be randomly assigned to conditions. The current experiment used a 2 (Language complexity: Simple vs. Complex) X 3 (Preference-consistency: Consistent, Inconsistent, No Preference) X 2 (Issue: Climate Change, Taxing Corporations) between subjects design. Because the primary analysis tests the interaction between complexity and preference-consistency, we collapsed across the two issues (however, exploratory analyses examined the effect of issue, as described below). The survey software was programmed to randomly assign participants to one policy that may be consistent, inconsistent, or neither (i.e. when participants have no policy preference) with their position on that issue. The policy was presented in either complex or simple language. Participants then rated their attitude toward the policy.

\section{Materials}

Attrition remedy. First, participants read a general description of the study:

"Thank you for choosing to participate in this HIT! In this study we will ask about your opinions on two political issues. We want to know what you think regardless of your level of interest or participation in politics. There are no right or wrong answers. We just want to know your honest opinions. Thank you!" Then participants completed a modified version of Zhou and Fishbach's (2016) attrition remedy on the next page, “Many mTurk workers tend to quit once they see the tasks. If a sizable number of people quit the survey halfway, the data quality of that survey would be compromised. However, our research depends on good quality data. Thus, before taking this survey, please make sure you do not mind completing the tasks described above." Then participants 
were instructed, "Please type below 'I will complete the full survey' so that we can ensure your data will be of high quality. Thank you!" and then there was a text box for them to type the requested sentence and another that asked for their mTurk identification number.

Policy preferences. Participants then rated their initial policy preferences by selecting either For, Against, or I Don't Know for both policies: “Are you for or against legislation that would require corporations to pay higher taxes?"; "Are you for or against legislation that would require businesses to report their usage of fuel and energy?" Participants also rated the personal importance of each issue $(1=$ Not at all important, 7 $=$ Extremely important $)$.

Attention check. To ensure participants were not mindlessly responding, we included a modified attention check question from Oppenheimer, Meyvis, and Davidenko (2009). Participants were shown a block of text that appeared to be a question about the kinds of political activities in which the participants engages. After three sentences, the message instructed the participant to ignore the instructions and click on an option they would otherwise never choose and ignore all other options ("Run for president of the USA"; see Appendix B). Hauser and Shwarz (2016; Study 1) found that 95\% of mTurk workers passed a similar attention check whereas only $39 \%$ of subject pool participants did. This supported our decision to recruit our participants from mTurk.

Preference-consistency manipulation. Belief-consistency was manipulated by randomly assigning participants to a policy that was either "for" or "against" one of the initial issues participants rated in the beginning of the experiment. We used participants' initial policy position (i.e. For, Against, or I Don't Know) to categorize them as having 
received a preference-consistent policy (for a policy they are for, or against a policy they are against), a preference-inconsistent policy (against a policy they are for, or for a policy they are against), or neither (when they marked I Don't Know as their initial position; see section below on Pre-processing steps).

Language complexity manipulation. Issue complexity was manipulated by presenting the policy in either simple or complex language. Simply worded policies were revised using a thesaurus and online dictionaries to replace simple words with complex words. For example, the simply worded policy against environmental restrictions on business (i.e. against addressing climate change) was "This initiative is to loosen restrictions on businesses that suffer due to the spread of climate change information. They will not have to report their usage of any form of fuel and energy." The complex version of this policy was "This initiative is to slacken circumscriptions on vendors that have been writhing due to propagation of ecological data. They will not have to report their expenditure of materials that lead to radiative forcing such as Chlorofluorocarbons."

To ensure that the complex policies were more complex than the simple policies, we submitted the policies to the Flesch-Kincaid readability test (Flesch, 1948) that computes the ease-of-readability of a passage based on the total number of words, sentences, and syllables in the passage. Complex policies had a much lower score $(M=$ 21.7) than simple policies $(M=56.1)$, indicating they were more difficult to understand. All versions of the policy proposals used in this experiment- along with their corresponding Flesch-Kincaid readability test scores - are available in Appendix C.

We sought to corroborate our readability analysis of complexity by pretesting the processing ease of all policies in the experiment. We recruited 65 participants from 
Amazon's Mechanical Turk and randomly assigned them to rate either the four complex (For and Against for each issue) or four simple versions of the policies ${ }^{2}$. Participants read "While ignoring your own opinion on the message content, please rate your agreement with each statement regarding each brief message. The questions aim to measure how complex the wording is for each message" and then rated the four policies on processing ease (see question in the section below). We conducted independent samples t-tests to determine if there were significant differences in processing ease between policies with simple versus complex language. In all cases, participants in the simple condition rated each policy as significantly easier to process than participants in the complex condition (all $p$ s $<.001 ;$ see Table 1). Further, we used a mixed ANOVA to test the 2 (Language complexity: Simple vs. Complex) X 4 (Issue: Climate Change Against, Climate Change For, Taxing Corporations Against, Taxing Corporations For) interaction to ensure that differences in processing ease for simple versus complex policies do not differ as a function of which policy the participant was rating. The interaction was not significant, $\mathrm{F}(3,180)=.32, p=.812$. Further, we collapsed policies across issue position (i.e. For vs. Against) and conducted paired samples t-tests to determine if there were differences in processing ease within complex and simple conditions. The difference in processing ease was not significant within either the complex condition, $t(30)=1.67, p=.105$, or within the simple condition, $t(30)=1.57, p=.127^{3}$.

\footnotetext{
2 Three participants were removed from the pretest because of missing data.

${ }^{3}$ It is worth noting that both t-tests are not very far from conventional levels of statistical significance and are both in the same direction, showing that policies on taxing corporations were somewhat easier to process than policies on climate change. Because the differences are not substantial, we argue that it is sufficient to test if issue moderates the expected 2 (Language complexity: Simple vs. Complex) X 3 (Preference-consistency: Consistent vs. Inconsistent vs. No preference) interaction.
} 
Processing ease. The mechanism of processing ease was measured with one item: "I can easily understand how this policy works" $(1=$ strongly disagree to $7=$ strongly agree; Shepherd \& Kay, 2012; Shockley \& Fairdosi, 2015).

Policy attitude. People's attitude towards the policy was assessed with three items adapted from Shockley and Fairdosi (2015): "Do you like or dislike the effect of this policy on businesses?"; "Do you like or dislike the idea of this policy being put into effect?" (1 = strongly dislike, 7 = strongly like); "Would you vote in favor or against this policy?" ( 1 = definitely vote against, 7 = definition vote in favor $)$. The items were the same for both issues. All items were averaged to form an attitude favorability composite $(\alpha=.97)$. Attitude favorability was the primary dependent measure.

\section{Results}

\section{Pre-processing steps}

Because our main analysis depends on participants' initial views on political issues, we first created our preference-consistency variable. For example, if a participant was in favor of taxing corporations and received a policy that argued in favor of that position, that variable was coded as preference-consistent. If they were in favor of taxing corporations but receive a policy that argues against that position, that variable was coded as preference-inconsistent. People who marked "I Don't Know" on their assigned issue were coded as no-preference. The same was applied to both issues.

\section{Preregistered analyses}

Initial analyses. We conducted an independent samples t-test to test if simply worded policies were rated as easier to process than complexly worded policies (i.e. manipulation check). Indeed, participants rated policies using simple language as 
significantly easier to process $(M=4.98, S D=1.81)$ than policies using complex language $(M=3.26, S D=1.80), t(319)=8.50, p<.001, d=.95,95 \%$ CI $[1.38,2.05]$ (one-tailed).

We conducted a between-participants ANOVA to test the 2 (Language complexity: Simple vs. Complex) X 3 (Preference-consistency: Consistent, Inconsistent, or No preference) X 2 (Issue: Climate Change vs. Taxing Corporations) interaction to determine if issue moderated our primary analysis of interest (the Language Complexity X Preference Consistency interaction). As expected, the three-way interaction was not significant, $\mathrm{F}(2,308)=.60, p=.552, \eta^{2}$ partial $=.004$ (two-tailed). Because we expected the effects of complexity to reverse depending on whether the policy was consistent or inconsistent with participants' initial policy preference, we did not expect a main effect of complexity. However, the main effect of complexity was significant, $\mathrm{F}(1,308)=5.12, p$ $=.024, \eta_{\text {partial }}^{2}=.016$ (two-tailed). In line with previous research (Shockley \& Fairdosi, 2015), participants rated policies using simple language more favorably $(M=4.08, S D=$ 2.20) than policies using complex language $(M=3.84, S D=1.61)$. As expected, there was a significant main effect of belief consistency, $\mathrm{F}(2,308)=76.23, p<.001, \eta_{\text {partial }}^{2}=$ .331 (one-tailed). Independent samples t-tests demonstrated that participants rated the policy consistent with their preference significantly more favorably $(M=4.97, S D=$ 1.65) than when it was inconsistent with their policy preference $(M=2.84, S D=1.65)$, $t(278)=10.83, p<.001, d=1.30,95 \%$ CI $[1.81,2.46]$ (one-tailed), or when the participant indicated no position on the issue (i.e. answered "I Don't Know") $(M=4.05$, $S D=1.50), t(180)=3.34, p<.001, d=.62,95 \%$ CI $[.49,1.45]$ (one-tailed). Additionally, participants rated a policy on which they had no preference significantly more favorably 
than a policy that was inconsistent with their preference, $t(176)=4.04, p<.001, d=.75$, $95 \%$ CI $[.69,1.64]$ (one-tailed). Unexpectedly, there was a main effect of issue such that participants ratings on corporate taxes were significantly more favorable $(M=4.09, S D=$ 1.78) than ratings on climate change $(M=3.79, S D=2.02), \mathrm{F}(1,308)=5.09, p=.025$, $\eta_{\text {partial }}^{2}=.016$ (two-tailed). Also unexpected, the complexity X issue interaction was also significant such that the simple effect of complexity on attitudes toward corporate taxes was significant, but the simple effect of complexity on attitudes toward climate change was negligible, $\mathrm{F}(1,308)=7.51, p=.006, \eta_{\text {partial }}^{2}=.024$ (two-tailed).

Tests of main hypotheses. As per our preregistered hypothesis, we expected that when participants receive an issue that is consistent with their policy preference, they would rate it more favorably when it was simply worded than when wording was complex. We expected the same effect for when participants rate an issue on which they have no policy preference. However, we hypothesized that when participants rate a policy that is inconsistent with their policy preference, they will rate it less unfavorably when it is complex than when it is simple (see Figure 1 for our preregistered proposed results). Because the 2 (Language Complexity) X 3 (Belief Consistency) X 2 (Issue) three-way interaction was not significant, we collapsed across issue and conducted a betweensubjects ANOVA to test the 2 (Language Complexity) X 3 (Belief-Consistency) interaction - our main analysis of interest. As expected, it was significant, $\mathrm{F}(2,314)=$ $15.51, p<.001, \eta_{\text {partial }}^{2}=.090$ (one-tailed; see Figure 2). To parse the interaction we conducted three independent samples t-tests examining whether language complexity caused significant differences in policy favorability separately for each level of beliefconsistency. As expected, when the policy was consistent with participants' policy 
preference, policies using simple language were rated significantly more favorably $(M=$ $5.78, S D=1.35)$ than those using complex language $(M=4.42, \mathrm{SD}=1.62), t(138)=$ $5.24, p<.001, d=.92,95 \%$ CI $[.93,1.80]$ (one-tailed). Likewise, when participants indicated that they had no policy preference, policies using simple language were rated significantly more favorably $(\mathrm{M}=4.44, \mathrm{SD}=1.76)$ than those using complex language $(\mathrm{M}=3.68, \mathrm{SD}=1.12), t(43)=1.73, d=.51, p=.046,95 \% \mathrm{CI}[.02,1.50]$ (one-tailed). Also consistent with our hypothesis, when participants rated a policy that was inconsistent with their initial policy preferences, they rated the policy less unfavorably when it contained complex language $(M=3.19, S D=1.51)$ than when it contained simple language $(M=2.48, S D=1.72), t(133)=-2.57, p=.006, d=-.44,95 \%$ CI $[-1.17$, -.25] (one-tailed).

Mediation analyses. Next, as outlined in our preregistered analysis plan, we sought to test if processing ease mediated the effect of language complexity on policy favorability. We used the PROCESS macro in SPSS (Model 4; Hayes, 2013) to run a simple mediation model separately for each level of belief-consistency (i.e. consistent, inconsistent, or no preference). In each model, we entered language complexity as the predictor $(\mathrm{X} ;$ simple $=0$, complex $=1)$, processing ease as the mediator $(\mathrm{M}$; continuous $)$, and policy favorability as the dependent variable (Y; continuous) (see Figure 3). As expected, when the policy was consistent with participants' preference, complexity led to lower policy favorability indirectly through processing ease as evidenced by the bootstrap confidence interval that excludes zero (see Table 2 for all mediation results). The results were the same for participants that received a policy on which they had no preference. When the policy was consistent with participants' preference, complexity led to higher 
policy favorability indirectly through processing ease, but the bootstrap confidence interval overlapped with zero and is therefore not significant.

Exploratory analyses. We were interested in testing whether our main analysis of interest (the complexity $\mathrm{X}$ belief-consistency interaction) would be moderated by issue importance. We marked this analysis as exploratory because we were able to come up with rationale for expecting that the interaction would be either stronger or weaker for issues that were rated as more important. For example, it is plausible that the effect size for issues rated as most important will be larger because when it is described in simple language, participants should be more inclined to give it an extreme rating, thus leading to a larger difference between complexly versus simply worded policies. However, it is also plausible that the effect size will be smaller for issues rated as most important. Important issues should be seen as personally relevant, which has been repeatedly found to increase elaboration of the message content (Petty, Cacioppo, \& Goldman, 1981; Petty \& Cacioppo, 1986). Thus, such close attention to highly important policies may reduce the effect of complexity on policy attitude. Because issue importance could produce plausible effects in opposite directions, it was difficult to make predictions and this analysis was therefore considered exploratory.

In order to explore the potential moderating effects of issue importance, we entered Language Complexity, Issue Importance, and two dummy variables for Belief Consistency (reference category $=$ No preference) into a multiple regression model. Issue importance did not moderate Complexity X Belief-Consistency interaction as indicated by the non-significant three-way interaction, $(b=-.16, S E=.21), t(309)=-.77, p=.444$, 95\% CI $[-.57, .25]$ (two-tailed). The Belief-Consistency X Issue Importance interaction 
was significant, $(b=.40, S E=.11), t(309)=3.63, p<.001,95 \%$ CI $[.18, .61]$ (twotailed). Unsurprisingly, the effect of belief-consistency (i.e. belief-consistent policies rated more favorably than belief-inconsistent policies) got stronger as issue importance increased, as denoted by the significant positive interaction term.

\section{Discussion}

Our results confirm our primary hypothesis that belief-consistency moderates the effect of language complexity on policy favorability. That is, when participants received a policy that was consistent with their beliefs, they rated it more favorably when it contained simple language than when it contained complex language. The effect was the same when participants had no policy preference, consistent with the prior literature on the effect of language complexity that had not considered the role of pre-existing opinions. On the other hand, when participants received a policy that was inconsistent with their beliefs, they rated it less unfavorably when it contained complex language than when it contained simple language. We also confirmed that processing ease mediated the effect of language complexity on policy favorability when participants were rating either a belief consistent policy or one in which they had no preference. However, processing ease did not mediate the effect of language complexity on policy favorability when participants received a belief-inconsistent policy. Additionally, neither issue (corporate taxes or climate change), nor issue importance moderated our effects.

These findings contextualize recent research that demonstrates that complex language causes lower policy favorability (Shockley \& Fairdosi, 2015). That is, we demonstrate that complexity lowers policy favorability only when the policy is consistent with the participant's initial policy preferences or if the participant has no initial policy 
preference. On the contrary, we demonstrate that the opposite occurs when the policy is inconsistent with participants' beliefs. In this case, participants rated a policy with complex language more favorably (or less unfavorably) than one using simple language. These results can be explained by Shah and Oppenheimer's (2007) framework on cue weighting. That is, information that is difficult to process (i.e. complex language) is weighed less heavily in attitude judgment than information that is easy to process (i.e. simple language). For example, complex policy language made it difficult for participants to process their favor (or disfavor) towards the policy, making their attitudes less favorable when they agreed with the policy and less unfavorable when they disagreed with the policy. In short, their attitudes became less extreme. These results extend previous research (Shah \& Oppenheimer, 2007) by incorporating people's pre-existing attitudes towards the attitude object as opposed to novel positivity or negativity of the information. Additionally, this extends the phenomenon of cue weighting to judgments of policy favorability.

It is worth noting the variability in effect sizes. According to our framework, it is plausible to expect that the difference in favorability between simple and complex policies would be larger when participants have a pre-existing position on the policy (preference-consistent or inconsistent) as opposed to when participants have no preference. However, this was apparent only for belief-consistent policies, in which the effect size was large. The effect size was actually smaller for belief-inconsistent policies than for those in which participants had no preference. It is unclear why the effect size was more than twice as large for belief-consistent policies than for belief-inconsistent policies. One possibility is that the differences in effect size were partly due to where 
favorability ratings were when the policy contained simple language. For preference consistent policies, the mean for the simply worded version was near the maximum value of the scale (i.e. 5.78; maximum =7). For preference-inconsistent policies, the mean for the simply worded version was not as extreme relative to the minimum value of the scale (i.e. 2.48; minimum =1). Because our results showed that people's favorability ratings became less extreme when the policy was shown in complex language, participants rating belief-inconsistent policies had a shorter distance to travel before they reached the midpoint (i.e. the place they presumably would not cross as a result of complexity alone). Although this may account for some difference in effect size, it is unlikely that it accounts for a substantial portion of the large difference in effect size we observed. A more plausible reason why the effect size was substantially smaller for belief-inconsistent policies is because belief-inconsistent information is subject to more scrutiny than beliefconsistent information (see Edwards \& Smith, 1996). Thus, this could reduce the effect of complexity because people are dedicating more cognitive resources to scrutinizing the belief-inconsistent policies, thereby making language complexity less influential in one's judgment. This might also explain why processing ease did not mediate the effect of complexity on policy favorability for belief-inconsistent policies but did for beliefconsistent policies and those on which participants had no preference.

\section{Limitations and future directions}

A potential criticism of this work is that the wording of the complex policies were inscrutable and therefore participants moved towards the center of the attitude scale because of their total lack of understanding. However, this is unlikely because there was a large main effect of belief-consistency, demonstrating that participants were in fact able 
to identify the policy as in line with their preferences when the policy was complex, they just did so less strongly.

Additionally, it is not totally clear how this research reconciles with Menegatti and Rubini's (2013) findings that abstract messages are more persuasive than concrete messages when the audience holds a similar position but concrete messages are more persuasive than abstract messages when the audience holds a different position from the speaker. Our experiment aimed to keep everything about each policy the same (including level of abstraction) except for our two independent variables: language complexity and issue-preference. Menegatti and Rubini (2013), suggest that abstract language is more persuasive to an audience with a similar position to the speaker because having common ground with the audience allows the speaker to rely more on figurative language. When the audience holds a different position from the speaker or is heterogeneous, the speaker has to bridge a gap in common knowledge and is therefore more persuasive when they use concrete language. In our current experiment, all language was relatively concrete. However, it would be interesting for future research to examine if the effect of language complexity is different depending on the level of language abstraction.

Another limitation of this work is that we did not manipulate conceptual fluency. That is, we manipulated language complexity while keeping the policy concepts conceptually the same. For example, a conceptually complex policy would have many working parts whereas a conceptually simple policy would have few. It is plausible to expect that conceptual fluency works similarly to language complexity in that it makes people weigh complex concepts less heavily in their judgments. A potentially fruitful way to explore this would be to manipulate the conceptual fluency of several issues and 
gauge the importance of each issue participants assign to conceptually simple (i.e. fluent) versus complex (i.e. disfluent) issues. Consider, for example, the issue of healthcare in the United States. The same issue could be framed as relying on few factors (e.g. whether our government decides to allocate the funds to it) or a collection of factors (e.g. whether states decide to opt in, cutting funds from other programs, and projections of cost). Additionally, it would be possible to use the same manipulation between issues. For example, when people decide how much weight they place on a particular issue when evaluating political candidates, they might place greater weight on issues that (at least seemingly) rely on fewer factors (i.e. conceptually simple) than those that rely on several factors (i.e. conceptually complex).

Another interesting future direction for this research would be to investigate the role of complexity (and thus processing fluency) in defense of one's policy positions. For example, in her investigation of the Clinton-Lewinsky affair, Ahluwalia (2000) found that when the information against then-president Bill Clinton was too difficult to refute, Clinton supporters defensively reduced the weight they placed on negatively affected traits (e.g. honesty) and raised the weight they placed on other traits such as Clinton's intelligence. It is plausible to expect that people defensively shift their reasons for supporting a political candidate (i.e. the weight they place on a given trait or issue) towards traits that easily come to mind (high accessibility) or traits that are otherwise easy to process (high conceptual fluency). Because our findings demonstrate that people weigh information more heavily when it is easy to process, people who are motivated to maintain a positive view of their favored political candidate can defend their support for the candidate by shifting the weight they assign to criticized issues and move the weight 
towards issues that are easy to process (e.g. has high accessibility, relies on few factors, or if person has ample information on the issue).

In conclusion, communication of policy information is a critical factor in determining people's attitudes towards a policy. It is worrisome that wording differences can have substantial effects on policy attitudes because "muddying the waters" (i.e. making an issue more confusing) can become the tactic of unscrupulous political partisans as a way of reducing support for a policy they are against. On the other hand, simplicity of policy information can help garner support for important policies. The current work sheds light on the importance of not only the content of a policy, but how it is communicated. 


\section{References}

Ahluwalia, R. (2000). Examination of psychological processes underlying resistance to persuasion. Journal of Consumer Research, 27(2), 217-232.

Alter, A. L., \& Oppenheimer, D. M. (2006). Predicting short-term stock fluctuations by using processing fluency. Proceedings of the National Academy of Sciences, 103(24), 9369-9372.

Cronin, T. E. (1989). Direct democracy: The politics of initiative, referendum, and recall. New York, NY: Harvard.

Edwards, K., \& Smith, E. E. (1996). A disconfirmation bias in the evaluation of arguments. Journal of Personality and Social Psychology, 71(1), 5.

Everson, D. (1981). The effects of initiatives on voter turnout: A comparative state analysis. Western Political Quarterly, 34, 415-425.

Flesch, R. (1948). A new readability yardstick. Journal of Applied Psychology, 32(3), 221.

Gervais, W. M., \& Norenzayan, A. (2012). Analytic thinking promotes religious disbelief. Science, 336(6080), 493-496.

Hafer, C. L., Reynolds, K. L., \& Obertynski, M. A. (1996). Message comprehensibility and persuasion: Effects of complex language in counterattitudinal appeals to laypeople. Social Cognition, 14(4), 317.

Hauser, D. J., \& Schwarz, N. (2016). Attentive Turkers: MTurk participants perform better on online attention checks than do subject pool participants. Behavior research methods, 48(1), 400-407.

Hayes, A. F. (2013). Introduction to mediation, moderation, and conditional process 
analysis: A regression-based approach. New York: Guilford Press.

Magleby, D. B. (1984). Direct legislation: Voting on ballot propositions and the United States. Baltimore, MD: Johns Hopkins.

Oppenheimer, D. M. (2006). Consequences of erudite vernacular utilized irrespective of necessity: Problems with using long words needlessly. Applied Cognitive Psychology, 20(2), 139-156.

Oppenheimer, D. M., Meyvis, T., \& Davidenko, N. (2009). Instructional manipulation checks: Detecting satisficing to increase statistical power. Journal of Experimental Social Psychology, 45(4), 867-872.

Petty, R. E., \& Cacioppo, J. T. (1986). The elaboration likelihood model of persuasion. In Communication and persuasion (pp. 1-24). Springer New York.

Petty, R. E., Cacioppo, J. T., \& Goldman, R. (1981). Personal involvement as a determinant of argument-based persuasion. Journal of personality and social psychology, 41(5), 847 .

Preacher, K. J., \& Hayes, A. F. (2004). SPSS and SAS procedure for estimating indirect effects in simple mediation models. Behavioral Research Methods, Instruments, \& Computers, 36, 717-731.

Preacher, K. J., \& Hayes, A. F. (2008). Asymptotic and resampling strategies for assessing and comparing indirect effects in multiple mediator models. Behavioral Research Methods, 40, 879-891.

Reber, R., Schwarz, N., \& Winkielman, P. (2004). Processing fluency and aesthetic pleasure: Is beauty in the perceiver's processing experience? Personality and Social Psychology Review, 8, 364-382. 
Shah, A.K. and Oppenheimer, D.M. (2007) Easy does it: The role of fluency in cue weighting. Judgment and Decision Making, 2, 371-379.

Shepherd, S., \& Kay, A. C. (2012). On the perpetuation of ignorance: System dependence, system justification, and the motivated avoidance of sociopolitical information. Journal of Personality and Social Psychology, 102(2), 264-280.

Shockley, E., \& Fairdosi, A. S. (2015). Power to the People? Psychological Mechanisms of Disengagement From Direct Democracy. Social Psychological and Personality Science, 6(5), 579-586. 


\section{Appendix A}

\section{G*Power analysis}

F tests-ANOVA: Fixed effects, special, main effects and interactions

Analysis: A priori: Compute required sample size

Input:

Effect size $f$

$\alpha$ err prob

Power ( $1-\beta$ err prob)

Numerator df

Number of groups

Output:
Noncentrality parameter $\lambda$ Critical $\mathrm{F}$ Denominator $\mathrm{df}$ Total sample size

Actual power
$=.1825$

$=0.05$

$=.8$

$=2$

$=12$

$=9.7587312$

$=\quad 3.0278979$

$=281$

$=293$

$=0.8008869$ 


\section{Appendix B}

\section{Attention check}

Individual preferences and situational variables can greatly impact decision processes. In order to facilitate our research on decision-making we are interested in knowing certain factors about you, the decision maker. Specifically, we are interested in whether you actually take the time to read the directions; if not, then some of our manipulations that rely on changes in the instructions will be ineffective. So, in order to demonstrate that you have read the instructions, please select the box labeled "Run for President of the USA" and ignore all of the other choices. Then move on to the next page. Thank you very much.

Which of these activities do you engage in regularly? (select all that apply)

- Protest

- Sign petitions

- Call representative

- Riot

- Boycotting

- Run for President of the USA 


\section{Appendix C}

Policies (readability scores were not displayed in the experiment)

\section{Climate change policies}

Simple/For. This initiative is to tighten restrictions on businesses whose practices are contributing to climate change. They will have to report their usage of all forms of fuel and energy. (Flesch-Kincaid readability score $=55.0$ )

Simple/Against. This initiative is to loosen restrictions on businesses that suffer due to the spread of climate change information. They will not have to report their usage of any form of fuel and energy. (Flesch-Kincaid readability score $=56.7$ )

Complex/For. This initiative is to upsurge mandates on vendors that are degrading the environment. They will have to report their expenditure of materials that lead to radiative forcing such as Chlorofluorocarbons. (Flesch-Kincaid readability score = 30.8)

Complex/Against. This initiative is to slacken circumscriptions on vendors that have been writhing due to propagation of ecological data. They will not have to report their expenditure of materials that lead to radiative forcing such as Chlorofluorocarbons. $($ Flesch-Kincaid readability score $=26.4)$

\section{Taxing corporations policies}

Simple/For. This initiative seeks to raise taxes for corporations whose earnings are above a set dollar amount. The money collected from such a tax will be used to pay for government programs for the nation's citizens. (Flesch-Kincaid readability score = 56.1) 
Simple/Against. This initiative seeks to lower taxes for corporations whose earnings are above a set dollar amount. The money saved from such a tax reduction will be used to expand businesses to create jobs. (Flesch-Kincaid readability score $=56.7$ )

Complex/For. This initiative seeks to augment corporate tariffs on capital gains that occupy tariff cohorts exceeding fixed remuneration sums. The tariff monies collected will be utilized to subsidize government programs for national occupants. (FleschKincaid readability score $=16.1$ )

Complex/Against. This initiative seeks to curtail corporate tariffs on capital gains that occupy tariff cohorts exceeding fixed remuneration sums. The tariff monies averted will be utilized to subsidize commerce expansion to engender occupations. (FleschKincaid readability score $=13.4$ ) 
Tables

\begin{tabular}{lccccc}
\hline & \multicolumn{2}{c}{ Condition } & \multicolumn{2}{c}{$\begin{array}{c}\text { t-test complex vs. simple } \\
\text { (Positive t-value = simple is easier to process) }\end{array}$} \\
\hline \multicolumn{1}{c}{ Issue } & Complex & Simple & $\boldsymbol{t}$ & df & $\boldsymbol{p}$ \\
\hline Climate Change: Against & 3.23 & 5.32 & 4.16 & 60 & $<.001$ \\
Climate Change: For & 4.16 & 6.06 & 4.80 & 60 & $<.001$ \\
Corporate Taxes: Against & 3.90 & 6.00 & 4.78 & 60 & $<.001$ \\
Corporate Taxes: For & 4.42 & 6.13 & 4.16 & 60 & $<.001$ \\
\hline
\end{tabular}

Table 1. Mean pretest ratings $(\mathrm{N}=62)$ for processing ease $(1=$ strongly disagree, $7=$ strongly agree) where higher ratings indicate easier processing and independent samples t-tests demonstrating that simple versions of all policies, in all cases, are significantly easier to process. 


\begin{tabular}{|c|c|c|c|}
\hline & $\begin{array}{c}\text { Belief-Consistency } \\
\text { Condition }\end{array}$ & $\begin{array}{c}\text { Conditional } \\
\text { Direct Effects }\end{array}$ & Indirect Effects \\
\hline \multicolumn{4}{|l|}{$\mathbf{X} \rightarrow \mathbf{M}$} \\
\hline & Consistent & $-2.16[-2.73,-1.59]$ & \\
\hline & Inconsistent & $-1.28[-1.91,-.66]$ & \\
\hline & No Preference & $-2.13[-3.25,-1.01]$ & \\
\hline \multicolumn{4}{|l|}{$\mathbf{M} \rightarrow \mathbf{Y}$} \\
\hline & Consistent & $.52[.39, .64]$ & \\
\hline & Inconsistent & $-.05[-.20, .10]$ & \\
\hline & No Preference & $.37[.16, .59]$ & \\
\hline \multicolumn{4}{|l|}{$\mathrm{X} \rightarrow \mathrm{M} \rightarrow \mathrm{Y}$} \\
\hline & Consistent & & $-1.12[-1.62,-.70]$ \\
\hline & Inconsistent & & $.07[-.12, .32]$ \\
\hline & No Preference & & $-.79[-1.71,-.27]$ \\
\hline
\end{tabular}

Table 2. Mediation models using complexity $(\mathrm{X})$ to predict policy favorability $(\mathrm{Y})$ indirectly through processing ease (M). The same model was run separately for each level of belief consistency (consistent, inconsistent, and no preference). Numbers denote unstandardized coefficients with 95\% confidence intervals (bootstrapped CIs for indirect effects). Significant effects appear in bold. 
Figures

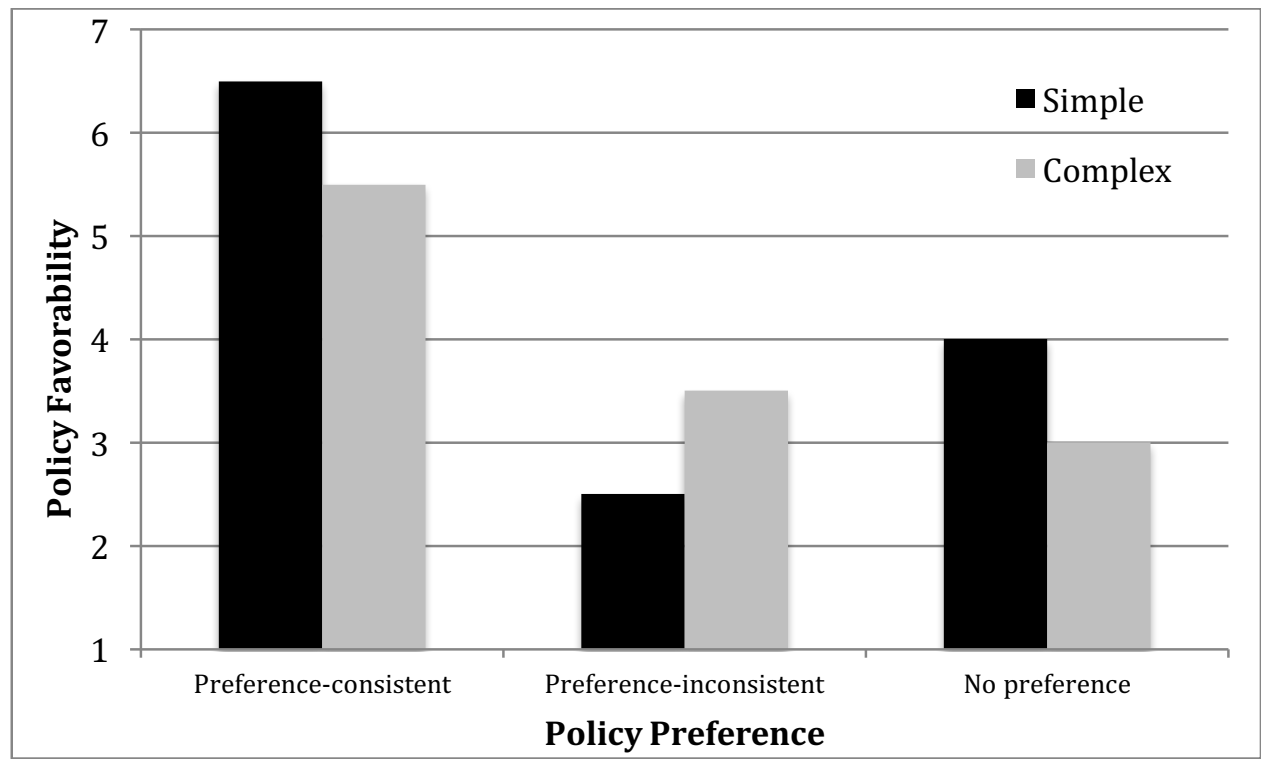

Figure 1. Preregistered figure of our proposed results for the effects of language complexity on policy favorability as a function of policy preference consistency. 


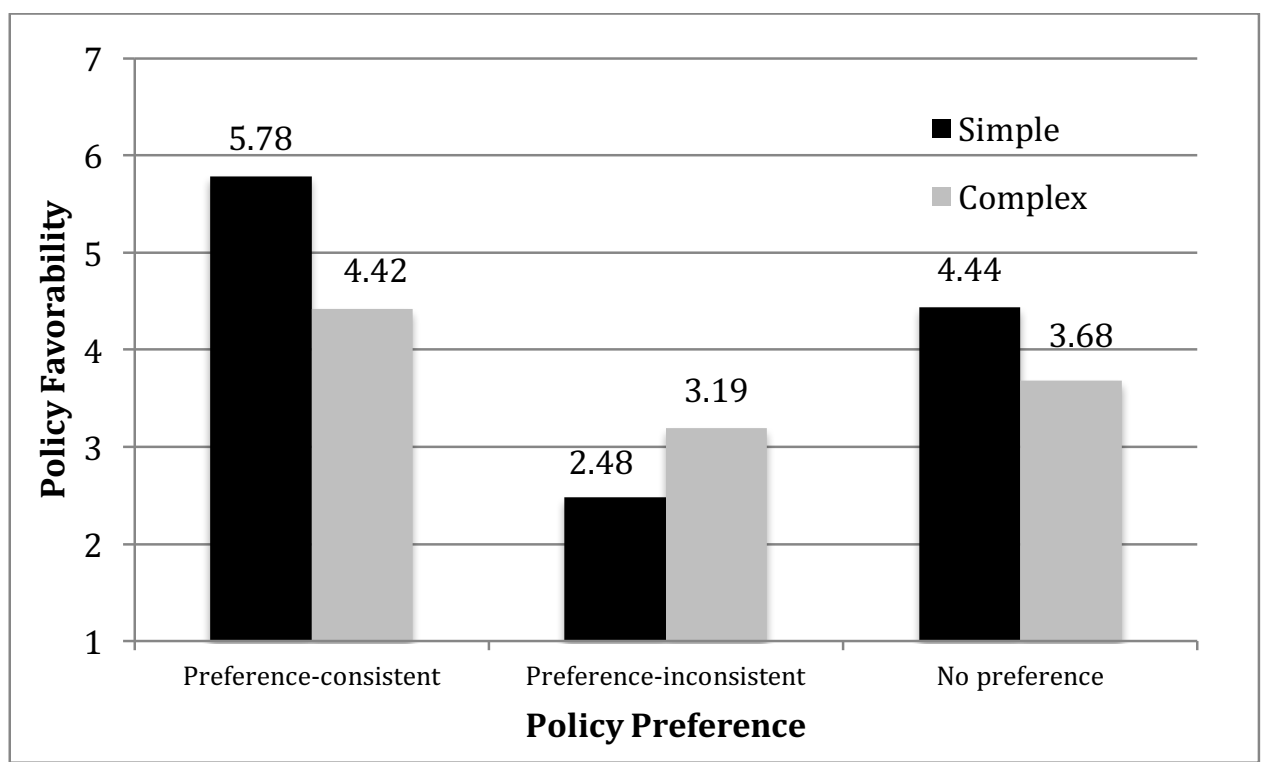

Figure 2. Observed results for the effects of language complexity on policy favorability as a function of policy preference consistency. 


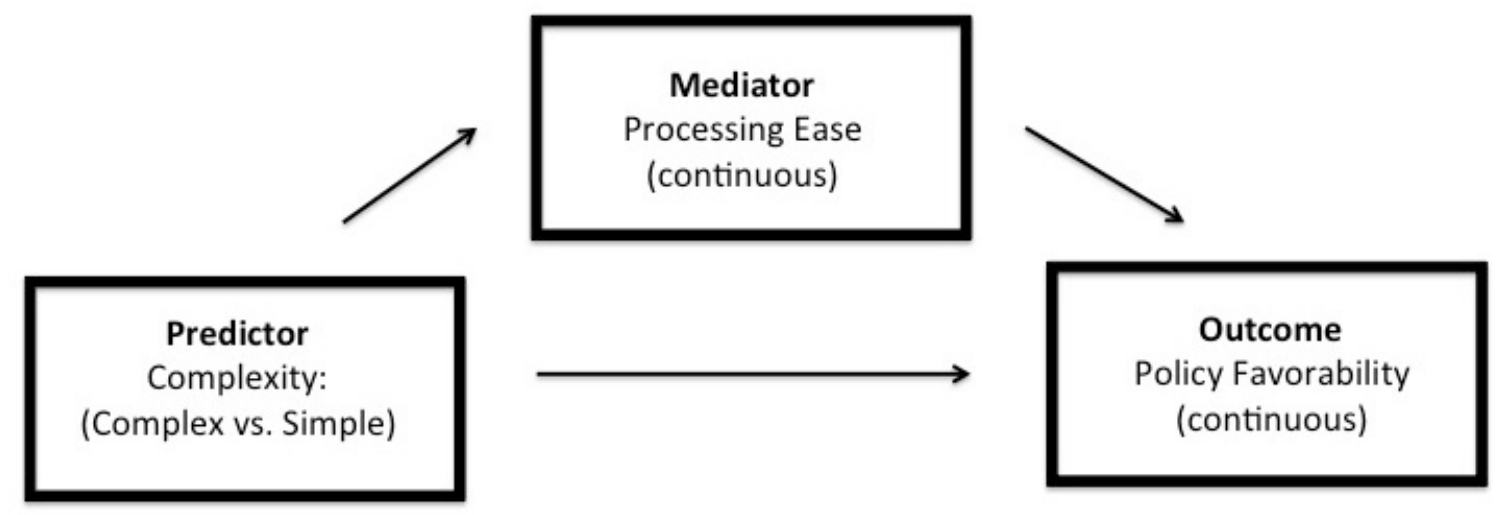

Figure 3. Mediation model testing if processing ease mediates the relationship between issue language complexity and policy favorability. We tested this model separately for each level of belief consistency (consistent, inconsistent, no preference). 